\title{
Myhre Syndrome
}

National Cancer Institute

\section{Source}

National Cancer Institute. Myhre Syndrome. NCI Thesaurus. Code C123815.

A rare, autosomal dominant inherited disorder caused by mutations in the SMAD4 gene.

It is characterized by developmental abnormalities, mild to moderate intellectual disability, hearing loss, skin stiffness, skeletal abnormalities, and typical facial features (short palpebral fissures, shortened distance between the nose and upper lip, midface hypoplasia, prognathism, cleft palate, and/or cleft lip). 